\title{
The Nosé-Hoover, Dettmann, and Hoover-Holian Oscillators
}

\author{
Wm.G. Hoover ${ }^{1 *}$ ，J.C. Sprott ${ }^{2}$ ，C.G. Hoover ${ }^{1}$ \\ ${ }^{1}$ Ruby Valley Research Institute \\ 601 Highway Contract 60 \\ Ruby Valley, Nevada 89833 \\ *E-mail: hooverwilliam@yahoo.com \\ ${ }^{2}$ University of Wisconsin-Madison \\ Department of Physics \\ Madison, Wisconsin 53706
}

Received: 10 July 2019; accepted: 27 July 2019; published online: 13 August 2019

\begin{abstract}
To follow up recent work of Xiao-Song Yang [1] on the Nosé-Hoover oscillator [2-5] we consider Dettmann's harmonic oscillator [6, 7], which relates Yang's ideas directly to Hamiltonian mechanics. We also use the Hoover-Holian oscillator [8] to relate our mechanical studies to Gibbs' statistical mechanics. All three oscillators are described by a coordinate $q$ and a momentum $p$. Additional control variables $(\zeta, \xi)$ vary the energy. Dettmann's description includes a time-scaling variable $s$, as does Nosé's original work [2,3]. Time scaling controls the rates at which the $(q, p, \zeta)$ variables change. The ergodic Hoover-Holian oscillator provides the stationary Gibbsian probability density for the time-scaling variable $s$. Yang considered qualitative features of Nosé-Hoover dynamics. He showed that longtime Nosé-Hoover trajectories change energy, repeatedly crossing the $\zeta=0$ plane. We use moments of the motion equations to give two new, different, and brief proofs of Yang's long-time limiting result.
\end{abstract}

Key words: Nosé-Hoover oscillator, Dettmann oscillator, Hoover-Holian oscillator, nonlinear dynamics

\section{BACKGROUND}

Nosé-Hoover dynamics was developed in 1984 as a side benefit of a Centre Européen de Calcul Atomique et Moléculaire ("CECAM") Workshop on Constrained Dynamics organized by Carl Moser. The workshop was held at Orsay, about 20 miles southwest of Paris. Shuichi Nosé and Bill Hoover met by chance at the Orly airport a few days prior to the Orsay meeting and were able to spend several hours together near the Notre Dame cathedral, discussing Nosé's recent work on the thermal control of molecular dynamics simulations $[2,3]$.

Seeking better to understand Nosé's innovative work Hoover applied Nosé's ideas to the one-dimensional harmonic oscillator problem [4-12]. He generated hundreds of solutions of the set of three ordinary differential equations using the fourth-order Runge-Kutta algorithm. The timedependent variables $(q, p, \zeta)$ are respectively the oscillator coordinate, momentum, and friction coefficient. Here are the three equations:

$$
\begin{aligned}
\{\dot{q}=p ; \dot{p}= & \left.-q-\zeta p ; \dot{\zeta}=\left[p^{2}-1\right] / \tau^{2}\right\} \\
& {[\text { Nosé-Hoover]. }}
\end{aligned}
$$

Provided that the mean value of the friction coefficient $\zeta$ is finite (so that the longtime average of its time derivative, $\langle\dot{\zeta}\rangle$, vanishes) the last equation implies that the mean oscillator temperature $\left\langle p^{2}\right\rangle$ is unity for long times. Thus $\zeta$ acts as a "thermostat". Posch, Hoover, and Vesely explored the detailed nature of the oscillator solutions [5] for a variety of response times $\tau$. Wang and Yang extended this work $[9,10]$ and found, in addition to the known periodic, toroidal, 
and fat-fractal chaotic trajectories, additional periodic trajectories, and tori, in the form of knots! The types of solutions found vary with $\tau$ and with the initial values of $(q, p, \zeta)$. In the present work we choose $\tau=1$.

\section{CONSEQUENCES OF TIME SCALING}

In the Summer of 1996 Carl Dettmann discovered a vanishing Hamiltonian which precisely reproduces the timedependence of the Nosé-Hoover flow where the NoséHoover momentum becomes $(p / s)[6,7]$.

$$
\begin{gathered}
\mathcal{H}_{\mathrm{D}}(q, p, s, \zeta) \equiv(s / 2)\left[q^{2}+(p / s)^{2}+\ln \left(s^{2}\right)+\zeta^{2}\right] \equiv 0 \rightarrow \\
\rightarrow s^{2}=e^{-\left[q^{2}+(p / s)^{2}+\zeta^{2}\right]}
\end{gathered}
$$

The "time-scaling variable" $s$ has the conjugate momentum $p_{s}=\zeta .|s|$ must be less than unity for $\mathcal{H}_{\mathrm{D}}$ to vanish. We select positive values, $0<s<1$, in our numerical work. The various resulting flows can occupy one-, two-, or threedimensional subspaces of the full stationary solution, the Gaussian function:

$$
(2 \pi)^{-3 / 2} \exp \left\{-\left[q^{2}+(p / s)^{2}+\zeta^{2}\right] / 2\right\} .
$$

With this Gaussian distribution known the normalized probability density for $s$ follows easily,

$$
\sqrt{(2 / \pi) \ln \left(s^{-2}\right)}
$$

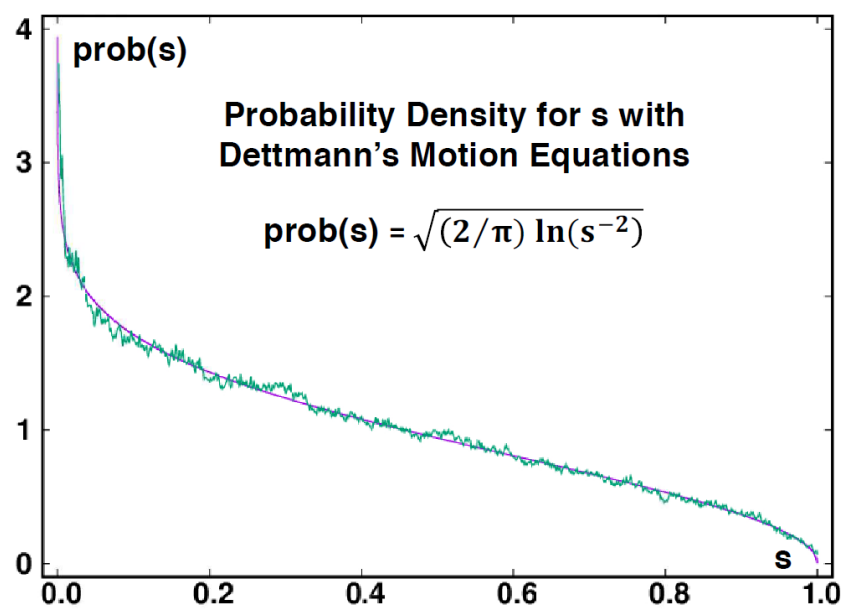

Fig. 1. Analytic and numerical probability densities for the timescaling variable $s$. Both are based on the three-dimensional Gaussian distribution with numerical values from the HooverHolian model

Fig. 1 compares this analytic distribution to a thousandpoint histogram from a fourth-order Runge-Kutta solution of the (ergodic) Hoover-Holian oscillator equations [8]:

$\left\{\dot{q}=p ; \dot{p}=-q-\zeta p-\xi p^{3} ; \dot{\zeta}=p^{2}-1 ; \dot{\xi}=p^{4}-3 p^{2}\right\}$.
For the Fig. $1 q$ and $p$ were initially unity and the two control variables were zero. Ten million timesteps, $d t=0.001$, were used. The stationary distribution for the Hoover-Holian equations is the four-dimensional Gaussian $(2 \pi)^{-2} \exp \left[\left(-q^{2}-p^{2}-\zeta^{2}-\xi^{2}\right) / 2\right]$. Because the model is ergodic, covering the entire four-dimensional Gaussian [8], we were able to use the three-dimensional Hoover-Holian subset $\left(q^{2}, p^{2}, \zeta^{2}\right)$ data as a model for the stationary Dettmann distribution $\left[q^{2},(p / s)^{2}, \zeta^{2}\right]$ with $s$ determined from the condition $\mathcal{H}_{\mathrm{D}} \equiv 0$. These numerical data, confirming our analytic work, are shown in Fig. 1.

Following up his earlier investigations of oscillator trajectories (including knots [9, 10]), Professor Xiao-Song Yang has recently proved that the oscillator motion, independent of the chosen initial conditions, must nearly always cross the $\zeta=0$ plane [1]. Yang considered the Nosé-Hoover equations, which follow from Dettmann's Hamiltonian with the replacement $(p / s) \rightarrow p$ :

$$
\left\{\dot{q}=p ; \dot{p}=-q-\zeta p ; \dot{\zeta}=p^{2}-1\right\} .
$$

The only situation in which the $\zeta=0$ plane is not crossed repeatedly is an unstable straight-line portion of the $\zeta$ axis:

$$
q(t)=q(0)=0 ; p(t)=p(0)=0 ; \zeta(t)=\zeta(0)-t .
$$

In the present work we first consider oscillator trajectories from the standpoint of the continuity equation in $(q, p, \zeta)$ space. Because the Nosé-Hoover equations are not strictly Hamiltonian, except in the $\mathcal{H}_{\mathrm{D}} \equiv 0$ case discovered by Dettmann, the Nosé-Hoover flow is compressible - the three-dimensional divergence is locally nonzero, responding linearly to the control variable $\zeta$ :

$$
\begin{gathered}
(\partial \dot{q} / \partial q)+(\partial \dot{p} / \partial p)+(\partial \dot{\zeta} / \partial \zeta)=0-\zeta+0 \\
{[\text { Dettmann }=\text { Nosé-Hoover }] .}
\end{gathered}
$$

Consider a longtime trajectory. Evidently a positive longtime average of $\zeta$ would correspond to a vanishing of the comoving volume and a dimensionality loss. A negative average would correspond to divergence, a numerical instability. In fact, the continuity equation has been used to explain the fractal nature of chaotic flows with positive friction [12].

In $(q, p, \zeta)$ space the continuity equation shows that the comoving volume element $\otimes$ vanishes and can become fractal if the longtime-averaged control variable is positive. The volume diverges, and the simulation stops, if that averaged variable is negative. The motion equations for the oscillator, in either $(q, p, \zeta)$ space or $\left(q, p, s, p_{s}=\zeta\right)$ space, can also be used to prove Professor Yang's zero-crossing result. We turn to that next.

\section{PROOFS OF PROFESSOR YANG'S $\zeta=0$ PLANE RESULT}

Yang's recent contribution [1] considers the threevariable Nosé-Hoover oscillator and shows algebraically that 
any longtime trajectory - other than the special constant $(q, p, \dot{\zeta})$ unstable straight line - must repeatedly cross the $\zeta=0$ plane. This is a handy result as the Poincare sections at that plane are commonly used to diagnose the fractal character of nonlinear flows. Yang's proof-of-crossing is relatively long, thirty pages, mainly algebra. Let us provide two simpler demonstrations of his result. Multiply the three motion equations by $q, p$, and $\zeta$ respectively and compute their longtime $(t \rightarrow \infty)$ averages $\langle\ldots\rangle$ in the usual way:

$$
\langle f[q, p, \zeta]\rangle=(1 / t) \int_{0}^{t \rightarrow \infty} f\left[q\left(t^{\prime}\right), p\left(t^{\prime}\right), \zeta\left(t^{\prime}\right)\right] d t^{\prime} .
$$

Assume also that $\left\langle q^{2}, p^{2}, \zeta^{2}\right\rangle$ are finite (so that their time derivatives average to zero). The three Nosé-Hoover differential equations then give three identities:

$$
\left\{\langle q p\rangle=0 ;\langle q p\rangle=\left\langle-\zeta p^{2}\right\rangle ;\langle\zeta\rangle=\left\langle\zeta p^{2}\right\rangle\right\}
$$

Combining the three shows that the mean value of $\zeta$ vanishes, equivalent to Professor Yang's result.

An even simpler demonstration follows from Nosé's original Hamiltonian [2, 3], $\mathcal{H}_{\mathrm{N}} \equiv(1 / s) \mathcal{H}_{\mathrm{D}}$, which gives a simple evolution equation for $s: \dot{s}=p_{s}$. Because $p_{s}$ and $\zeta$ are identical, as shown by Dettmann, the time-average of the $\dot{s}$ equation shows directly (assuming numerical convergence of the motion) that the control variable $\zeta$ has mean value zero, again implying Yang's result.

\section{AN AFTERWORD FOR YOUNG RESEARCHERS}

Lingering geometric and topological questions remain where the chaotic sea is concerned. The sea is an enduring paradoxical concept. Is it a set of three-dimensional points? Is it a continuum with holes here and there? Is it just a single chaotic trajectory? Is it an ill-defined limit? These questions remain at the research frontier. Let us have a look at the chaotic sea for the Nosé-Hoover oscillator.

Fig. 2 shows cross-sections of the three-dimensional oscillator's $(q, p, \zeta)$ phase space showing about ten million crossings of the three planes where each of the variables vanishes. The fourfold symmetry of these sections reflects the time-reversibility of the motion equations as well as the fact that any solution $\{+q,+p,+\zeta\}_{t}$ implies the existence of a mirror-image solution $\{-q,-p,+\zeta\}_{t}$, as well as $\{+q,-p,-\zeta\}_{t}$.

For $\tau=1$ about six percent of the Gaussian distribution [13] $e^{-\left(q^{2}+p^{2}+\zeta^{2}\right) / 2}$ appears to be a cohesive connected sea, penetrated by infinitely many regular orbits with zero Lyapunov exponents $\{\lambda\}$. These exponents describe the longtime average growth or decay rates of small perturbations. The boundary between points with a positive $\lambda$, charateristic of the sea, and points with all zero Lyapunov exponents is evidently murky and uncertain. The boundary region is likely fractal in the sense that in the neighborhood of any chaotic point in the sea there must be other points only a small distance away, located on periodic orbits of great length, well beyond our capacity to compute in any meaningful way.

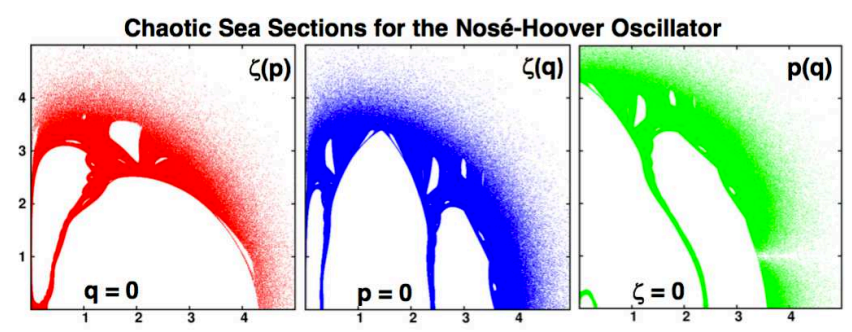

Fig. 2. Sections of the Nosé-Hoover Chaotic Sea where $q$ or $p$ or $\zeta$ vanishes. Only one quadrant is shown here as each of the Sections has fourfold symmetry

The mathematics of such sets of points is confused through concepts related to Hilbert's Hotel and the BanachTarski Theorem. Hilbert's Hotel, with positive integer room numbers, is alway full (with $\aleph_{0}$ guests) but with always an available room (by moving customers from $n$ to $n+1$ ). Similar mappings of points in two or three (or many) dimensional spaces enable the disection of a sphere into a finite number of pieces which can then be reassembled to make two spheres. This is an active field of mathematics at the moment. For a nicely illustrated description of the BanachTarski paradox see Ref. [14].

\section{Acknowledgment}

We thank Professor Yang for useful email communications and the anonymous referee for several useful suggestions which have improved this manuscript.

\section{References}

[1] X.S. Yang, Qualitative Analysis of the Nosé-Hoover Oscillator, submitted to Qualitative Theory of Dynamical Systems, 2019.

[2] S. Nosé, A Unified Formulation of the Constant Temperature Molecular Dynamics Methods, The Journal of Chemical Physics 81, 511-519 (1984).

[3] S. Nosé, A Molecular Dynamics Method for Simulations in the Canonical Ensemble, Molecular Physics 52, 255-268 (1984).

[4] Wm.G. Hoover, Canonical Dynamics. Equilibrium PhaseSpace Distributions, Physical Review A 31, 1695-1697 (1985).

[5] H.A. Posch, W.G. Hoover, F.J. Vesely, Canonical Dynamics of the Nosé Oscillator: Stability, Order, and Chaos, Physical Review A 33, 4253-4265 (1986).

[6] W.G. Hoover, Mécanique de Nonéquilibre à la Californienne, Physica A 240, 1-11 (1997).

[7] C.P. Dettmann, G.P. Morriss, Hamiltonian Reformulation and Pairing of Lyapunov Exponents for Nosé-Hoover Dynamics, Physical Review E 55, 3693-3696 (1997). 
[8] W.G. Hoover, B.L. Holian, Kinetic Moments Method for the Canonical Ensemble Distribution, Physics Letters A 211, 253-257 (1996).

[9] L. Wang, X.S. Yang, The Coexistence of Invariant Tori and Topological Horseshoes in a Generalized Nosé-Hoover Oscillator, International Journal of Bifurcation and Chaos 27, 1750111 (2017).

[10] L. Wang, X.S. Yang, Global Analysis of a Generalized NoséHoover Oscillator, Journal of Mathematical Analysis and Applications 464, 370-379 (2018).

[11] W.G. Hoover, J.C. Sprott, C.G. Hoover, A Tutorial. Adaptive Runge-Kutta Integration for Stiff Systems: Comparing the Nosé and Nosé-Hoover Oscillator Dynamics, American Journal of Physics 84, 786-794 (2016).

[12] W.G. Hoover, Computational Statistical Mechanics, Elsevier, New York (1991). Available free online at williamhoov er.info.

[13] P.K. Patra, W.G. Hoover, C.G. Hoover, J.C. Sprott, The Equivalence of Dissipation from Gibbs' Entropy Production with Phase-Volume Loss in Ergodic Heat-Conducting Oscillators, International Journal of Bifurcation and Chaos 26, 1-11 (2016).

[14] R. French, The Banach-Tarski Theorem, The Mathematical Intelligencer 10, 21-28 (1988).

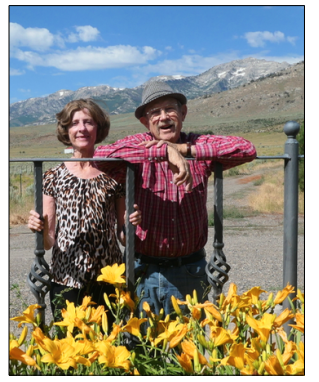

William G. Hoover, Carol G. Hoover; Bill and Carol Hoover retired to Ruby Valley Nevada in 2005 where they built a home and continued their research work in computational physics, chaos, and nonequilibrium systems through the magic of the Internet. The main business of the valley is raising beef, mainly Black Angus. The air is clean, the weather mild, and the general ambience well-suited to contemplation and creativity. The Hoovers have published four books since moving to the valley with the most recent, Microscopic and Macroscopic Simulation Techniques published by World Scientific in 2018, a summing up of 11 two-hour lectures presented to graduate students at the Indian Institute of Technology at West Bengal pursuant to the invitation of their good friend, Professor Baidurya Bhattacharya.

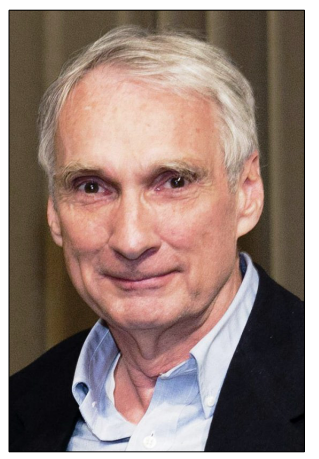

Julien Clinton Sprott received his $\mathrm{PhD}$ in physics from the University of Wisconsin in 1969 and joined the faculty there in 1973. After a 25-year career in experimental plasma physics, he became interested in computational nonlinear dynamics and chaos in 1988. He has authored or coauthored hundreds of papers on the subject and a dozen books. He is now Emeritus Professor of Physics at the University of WisconsinMadison. 